\title{
1D CNN-Based Intracranial Aneurysms Detection in 3D TOF-MRA
}

\author{
Wenguang Hou $\mathbb{D},{ }^{1}$ Shaojie Mei $\mathbb{D}^{1},{ }^{1}$ Qiuling Gui $\left(\mathbb{D},{ }^{1}\right.$ Yingcheng Zou $\mathbb{D},{ }^{1}$ Yifan Wang $\left(\mathbb{D},{ }^{1}\right.$ \\ Xianbo Deng $\mathbb{B}^{2},{ }^{2}$ and Qimin Cheng $\mathbb{D}^{3}$ \\ ${ }^{1}$ College of Life Science and Technology, Guangzhou, China \\ ${ }^{2}$ Department of Radiology, Union Hospital, Alberton, South Africa \\ ${ }^{3}$ School of Electronic Information and Communications, Huazhong University of Science and Technology, Wuhan 430022, China
}

Correspondence should be addressed to Xianbo Deng; 1951547885@qq.com and Qimin Cheng; 40328009@qq.com

Received 15 July 2020; Revised 30 September 2020; Accepted 31 October 2020; Published 12 November 2020

Academic Editor: Shafiq Ahmad

Copyright (c) 2020 Wenguang Hou et al. This is an open access article distributed under the Creative Commons Attribution License, which permits unrestricted use, distribution, and reproduction in any medium, provided the original work is properly cited.

\begin{abstract}
How to automatically detect intracranial aneurysms from Three-Dimension Time of Flight Magnetic Resonance Angiography (3D TOF MRA) images is a typical 3D image classification problem. Currently, the commonly used method is the Maximum Intensity Projection- (MIP-) based way. It transfers 3D classification into 2D case by projecting the 3D patch into 2D planes along different directions on the basis of voxel's intensity. After then, the 2D Convolutional Neural Network (CNN) is established to do classification. It has been shown that the MIP-based method can reduce the demands for the samples and increase the computation efficiency. Meanwhile, the accuracy is comparable with that of 3D image classification. Inspired by the strategy of MIP, we want to further reduce the demands for samples and accelerate the training by transferring the 2D image classification into 1D case, i.e., we want to generate the 1D vectors from the MIP images and then establish a 1D CNN to do intracranial aneurysm detection and classification for 3D TOF MRA image. Specifically, our method first extracts a series of patches as the Region of Interests (ROIs) along the blood vessels from the original 3D TOF MRA 3D image. The corresponding MIP images of each ROI will be obtained through maximum intensity projecting. Then, we generate a series of $1 \mathrm{D}$ vectors by accumulating each MIP image along different directions. Meanwhile, a $1 \mathrm{D} \mathrm{CNN}$ is established to detect aneurysms, in which, the input is the obtained $1 \mathrm{D}$ vectors and the output is the binary classification result denoting whether there are intracranial aneurysms in the considered patch. Generally, compared with 2D- and 3D-CNN, the 1D CNN-based way greatly accelerates the training and shows stronger robustness in the case of fewer samples. The efficiency of the proposed method outperforms the 2D CNN about 10 times in CPU training. Yet, their accuracies are close.
\end{abstract}

\section{Introduction and Motivation}

Intracranial Aneurysms (IA) are an important cause of high morbidity and mortality of cardiovascular diseases $[1,2]$. It has been reported that about $3 \%$ of healthy adults have Intracranial Aneurysms (IAs) [2]. Rupture of IAs is the major cause of subarachnoid hemorrhage, which often leads to severe neurological sequelae and even death [3]. Presently, Digital Subtraction Angiography (DSA) based on X-ray is still regarded as the golden standard in diagnosing IAs, since it can accurately reflect the location, scope, and degree of IAs. Its sensitivity is more than $95 \%[4,5]$. Nonetheless, DSA may lead to neurological problems due to its invasiveness
[6]. Different from DSA, Three-Dimension Time of Flight Magnetic Resonance Angiography (3D TOF MRA) has the advantages of noninvasive, radiation-free, and high sensitivity. As such, it has been widely used to detect aneurysms [7-9]. However, it is a challenging and laborious task for radiologists to detect the unruptured aneurysms through observing the MRA images frame by frame. Moreover, the sensitivity of manual diagnosis is only about $64-70 \%$ [10].

Computer Aided Diagnosis (CAD) has been widely employed to help doctors to do IAs $[1,11,12]$. The earlier CAD relied on the low-level or hand-crafted features. The effectiveness of these features depends on the specific domain knowledge of the designers. Generally, the results 
based on hand-crafted features are not robust and universal. To overcome the obstacle, the feature learning-based methods were proposed, which means that the feature detection algorithm is learned from the existing samples rather than being established by researchers in the domain. Recently, the Convolutional Neural Network (CNN) has been increasingly applied to the detection, classification, and segmentation of medical images [13-16]. The 3D CNNbased method utilizes the spatial structure information of volume and performs excellently through inputting the $3 \mathrm{D}$ image into CNN directly. Sichtermann established an IAs detection system on basis of a 3D CNN in [17]. This system utilized sufficient pretreatments and post-treatments to reduce the number of false positives and achieve high detection sensitivity. Allison used Computer Tomograph Angiography (CTA) images of the brain to construct a HeadXNet model to segment aneurysms [18], which predicted aneurysms with high sensitivity. Bio Joo conducted IAs detection based on the 3D ResNet leading to better result [19]. A cascade strategy was proposed to automatically detect Cerebral MicroBleeds (CMBs) from MR images using 3D CNN [20]. The detected CMBs in the second stage could be utilized as a reference for aneurysm detection. The 3D CNNbased methods could undoubtedly achieve excellent performance. However, the difficulty in obtaining lots of 3D medical images limits the practical applications. The Maximum Intensity Projection (MIP) strategy projects the original volume into $2 \mathrm{D}$ images to reduce the demands for samples in CNN-based detection and speed up the CNN training. Based on the above strategy, Nakao generated 9 MIP images and concatenated them into a new image as input and then do classification using 2D CNN in [21]. These MIP images should contain the main structural information of the original volume. As such, it can obtain the accuracy of $95 \%$, which is comparable to the 3D CNN-based method. Stember trained a U-net CNN with 250 MIP images to predict the size of aneurysms. Though its results are fine, but it is still limited in some special cases [22]. Duan proposed a secondary cascade CNN architecture to detect aneurysms [23]. Ueda utilized ResNet-18 to detect aneurysms, which had the sensitivity of $93 \%$ and $91 \%$ for internal and external datasets, respectively [24].

Compared with the 3D CNN-based method, the MIPbased methods greatly reduce the requirement for computer performance and the number of samples [21]. However, its demands are still considerable. Therefore, we intend to propose a new solution to convert 2D CNN-based method into the $1 \mathrm{D}$ case. The main idea is that the MIP image will be projected into some $1 \mathrm{D}$ vectors, and the $2 \mathrm{D} \mathrm{CNN}$ is also replaced by a $1 \mathrm{D}$ one. That is, we further reduce the dimension of the input data according to the way of generating MIP in classification accordingly. As such, this new strategy further reduces the redundant information and simplifies the structure of the network. In addition, the 1D CNN can be trained on the CPU while 2D or 3D CNN should be trained using GPU in general situation. It means that the demand for computer's performance is greatly reduced. As such, the contributions of this paper lie in the following. (1) The MIP strategy is extended to the $2 \mathrm{D}$ case, and $1 \mathrm{D}$ vectors are used to represent the $2 \mathrm{D}$ image which actually reduces the dimension of the original data. (2) Compared with the MIPbased method, the 1D CNN is more efficient because it requires fewer samples and shorter training time, while the accuracy is retained. (3) This way can be extended to other pattern recognition problems.

\section{Methodology}

2.1. The Basic Idea. The proposed strategy is illustrated as Figure 1. In 3D TOF MRA volume, we first extract a series of voxels along the cerebral vessel by thresholding the original $3 \mathrm{D}$ image. For each voxel, we segmented a 3D patch centered at that voxel from the 3D TOF MRA volume. The patch is called as the Region of Interest (ROI). For each ROI, some MIP images will be generated by projecting the ROI along several directions [21]. Note that MIP image contains the main features of the original volume. Accordingly, several $1 \mathrm{D}$ vectors (sizes are $m$ ) can be obtained by accumulating the pixels on the MIP image along different direction. Assume that we conduct the accumulation along $n$ directions. $n 1 \mathrm{D}$ vectors with size of $m$ can then be obtained for each ROI. Afterwards, these $1 \mathrm{D}$ vectors are concatenated into a new $1 \mathrm{D}$ vector whose length is $m * n$. In our experiment, we generate nine MIP images for each ROI. As such, there will be nine concatenated $1 \mathrm{D}$ vectors with length $m * n$ for each ROI. Then, these nine concatenated vectors are connected into a $9 * m * n$ 1D vector in Figure 1. Meanwhile, we establish a 1D CNN for aneurysm detection, whose input is a $9 * m * n 1 D$ vector and the output is 1 or 0 depending whether there is an aneurysm or not in the considered patch. After training the 1D CNN, we can use it to detect aneurysm from the TOF MRA volume. In short, we further project the MIP images into $1 \mathrm{D}$ vectors and establish the related $1 \mathrm{D} \mathrm{CNN}$ for aneurysm detection, which reduces the requirement for samples and improves the efficiency of training.

The direct way for IAs detection from 3D TOF-MRA image is to establish a $3 \mathrm{D} \mathrm{CNN}$, whose input is the original 3D ROI patch. The MIP-based method takes the several 2D images as the input and replaces $3 \mathrm{D} C \mathrm{CNN}$ with a $2 \mathrm{D}$ one which reduces the dimension of the input data and the amount of calculation. By comparison, 1D vectors, obtained from the MIP images shown in the bottom of Figure 1, are taken as the input of $1 \mathrm{D}$ CNN to do detection or classification. It is clear that the demands for samples are reduced and the training computations are lessened in case of $1 \mathrm{D}$ $\mathrm{CNN}$-based aneurysm detection. Meanwhile, the main characters of the original 3D patch are retained in the MIP images and the main characters of MIP images are remained in the generated $1 \mathrm{D}$ vectors. It means that the $1 \mathrm{D}$ vectors retain the main characters of the $3 \mathrm{D}$ patch, and the classification accuracy should be close to the $3 \mathrm{D}$ case.

2.2. Data Preprocessing. In generating the samples, some preprocessing operations are required for the original $3 \mathrm{D}$ TOF MRA volume. It is common that the volume may be anisotropic and the intensities of the volumes captured by different machines may be diverse. Therefore, resampling 


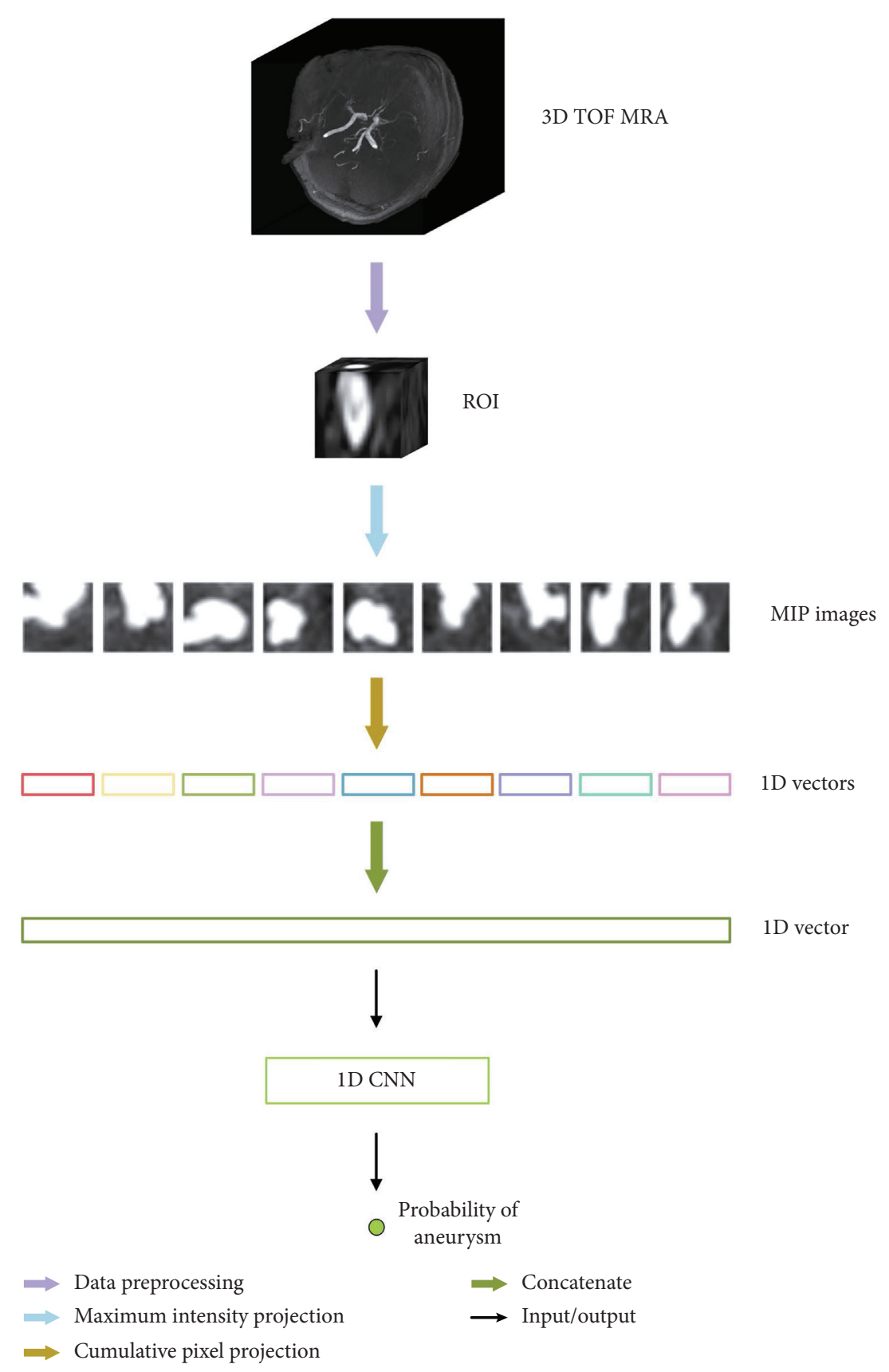

Figure 1: An overview framework for 1D CNN-based intracranial aneurysm detection.

and normalization are conducted for all slices of the volume. The classic bicubic interpolation algorithm is taken here to do resampling to obtain isotropic volume [25]. Meanwhile, we the grayscale stretch is applied to improve the homogeneity of these slices. We utilize a piecewise linear transformation function as equation (1) to make the gray value distribution be homogeneous:

$$
y= \begin{cases}\frac{(x-\min ) * b}{a-\min }, & x<a, \\ \frac{(255-b)(x-a)}{\max -a}, & x \geq a .\end{cases}
$$

In equation (1), $x$ and $y$ are the gray value of the original and stretched image, max and min denote the maximum and minimum gray of the original image, and parameters $a$ and $b$ determine the slope of the gray stretching.

IAs are attached to the blood vessels, which have obvious different intensity from other tissues in brain MRA image. Therefore, we can approximately separate the blood vessels from other tissues on the basis of a threshold. The OSTU and Hessian matrix are utilized to fulfill this task $[26,27]$. Then, we further judge whether there are IAs in the 3D TOF MRA data only taking the blood vessel into account. It should be noted that all positive samples contain at least one intracranial aneurysm, and all negative samples do not have one 
intracranial aneurysm. Then, a $3 \mathrm{D}$ patch of size $16 * 16 * 16$ centered at the voxel of the detected cerebral vessel is sliding along the vessels to generate a series of ROIs. A series of ROIs are produced while the patch center is sliding along the cerebral vessels. For all ROIs, these with intracranial aneurysms are taken as positive samples and the ROIs nearby the circle of Wills without intracranial aneurysms are considered as negative samples [28]. The process of obtaining the ROI is shown in Figure 2.

2.3. Generation of $1 D$ Vector. After data preprocessing, we obtain a series of normalized ROI, and we get nine MIP images for each ROI through nine projections [21]. For each MIP image, we adopt a new strategy called Pixel Accumulation Projection (PAP) to generate $1 \mathrm{D}$ vector by accumulating the pixels in a direction. It is clear that the generated $1 \mathrm{D}$ vectors will contain the main information of the corresponding MIP image. That is, the MIP image can be reconstructed by these projection vectors. The schematic and formula of the accumulation way are, respectively, shown in Figure 3 and equation (2). The MIP image is first expanded to its circumcircle by padding with zero. These zero pixels will not affect the accumulation value in generating $1 \mathrm{D}$ vector. Then, the circumcircle image is projected to obtain four $1 \mathrm{D}$ vectors along four different directions by accumulating the pixels as follows:

$$
y=\sum_{k=1}^{n} x_{k},
$$

where $n$ represents that there are $n$ pixels in the direction and $x_{k}$ denotes the value of the $k$ th pixel in the direction. It should be noted that the generated four vectors are resized to be 16 and then concatenated into a $641 \mathrm{D}$ vector. As such, nine MIP images mentioned above are further simplified into nine concatenated $1 \mathrm{D}$ vectors. The whole process of $1 \mathrm{D}$ vector generation is illustrated in Figures 4 and 5. As shown in Figure 6, the possibility of the considered MRA containing intracranial aneurysms will be less when the generated vector is smooth (the vector is shown as a curve), and vice versa. In our experiment, the original volume $\left(\right.$ shape $\left._{1}\right)$ is first projected into nine MIP images (shape $)_{2}$ ), which are further simplified into nine concatenated $1 \mathrm{D}$ vectors with the length of $64\left(\right.$ shape $\left._{3}\right)$. These nine $1 \mathrm{D}$ vectors also can be concatenated again to a $5761 \mathrm{D}$ vectors as $\left(\right.$ shape $\left._{4}\right)$. The sizes of different data are shown in Table 1 and Figure 5. shape ${ }_{1}$ is the $3 \mathrm{D}$ image and shape ${ }_{2}$ corresponds to the MIP image. shape ${ }_{3}$ denotes to the four vectors in middle of Figure 4 and shape ${ }_{4}$ means the concatenated vector with a size of 576 .

2.4. 1D Convolutional Neural Network. Based on the previous discussion, our proposed method is dedicated to reduce computation in training and the demand for samples. To verify the efficiency and accuracy of the proposed method, two 1D CNNs are used in our experiments. The overall structures of the two models are shown in Figures 7 and 8. The Model-1 is an 11-layer $\mathrm{CNN}$ which includes four convolutional layers, four maximum pooling layers, and three fully connected layers. Its input is a $1 \mathrm{D}$ vector with 576 elements, as shown in Figure 5. The Model-2 is a multichannel network, where nine inputs are connected to two fully connected layers via two convolutional layers and two maximum pooling layers. The parameters of the two models are listed in Tables 2 and 3, respectively.

In the convolutional layers, the network is designed to extract the aneurysm features, which is trained using the backpropagation algorithm. The weight-sharing mechanism is applied to the neurons located on the same feature map to reduce the risk of overfitting. The pooling mechanism is implemented by calculating the maximum or average value of the convolution features between adjacent neurons in the previous convolutional layers. The maximum pooling layers are utilized to reduce the dimension of feature extraction. We use several convolutional layers and maximum pooling layers to extract the features of $1 \mathrm{D}$ vectors. Finally, the output layer after the fully connected layers takes the softmax activation function to predict the probability of aneurysm. The binary crossentropy between expectation and reality is taken as the loss function in training the CNN. The binary crossentropy is

$$
L(p, t)=-[p \log (t)+(1-p) \log (1-t)]
$$

where $p$ is the prediction and $t$ denotes the label. To avoid overfitting, Batch Normalization (BN) is utilized to accelerate network convergence [29]. The Rectifier Linear Unit (ReLU) is then employed as the nonlinear activation function in the convolutional layer and fully connected layer [30]. The 1D convolution kernels are initialized as the way in [31]. ReLU can be denoted as follows:

$$
\operatorname{ReLU}(x)=\max (x, 0) .
$$

\section{Experiment Setup}

All experiments are implemented under the framework of Keras with TensorFlow, Windows machine. The CPU is Intel (R) Core (TM) i7-8700, and the GPU is GeForce GTX1050Ti with $4 \mathrm{~GB}$ memory. Data preprocessing is conducted on Matlab.

3.1. Dataset. All the tested datasets are sampled from the Union Hospital of Tongji Medical College and Huazhong University of Science and Technology from April 2017 to August 2019. These TOF MRA volumes are collected on two kinds of MRI machines with $1.5 \mathrm{~T}$ and $3.0 \mathrm{~T}$ magnetic intensity. After deleting the personal information of the patients, 164 cases were utilized for the experiments. It should be mentioned that each volume at least contains one unruptured intracranial aneurysm. These volumes are anisotropic. Then, we resample them to generate isotropic data using the bicubic interpolation algorithm. For all volumes, the average number of slices is 121 . The minimum and maximum values are 91 and 177, respectively. The average number of slices containing intracranial aneurysms is 8 , with a minimum of 3 and a maximum of 31 . These slices ranged in 


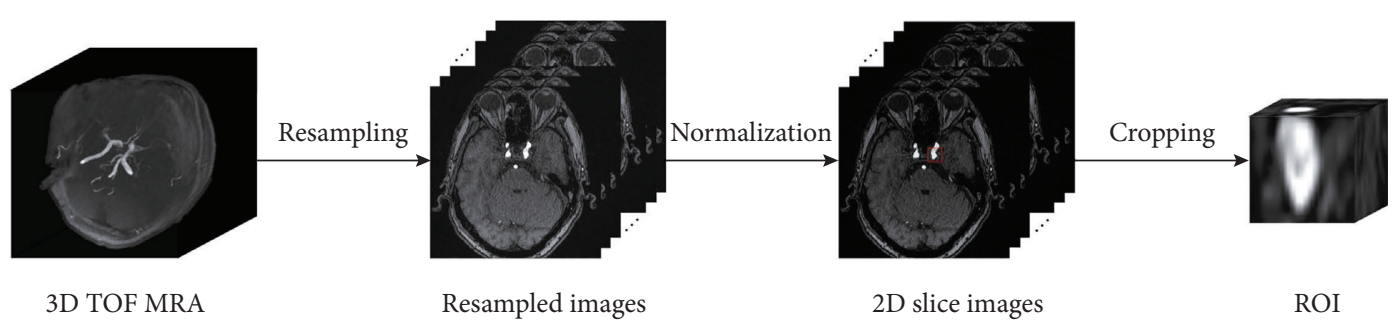

FIgURE 2: The flowchart of data preprocessing.

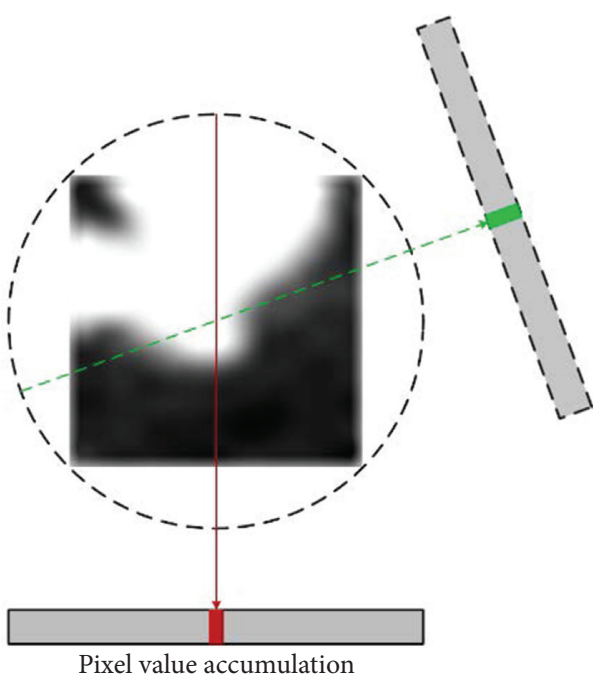

FIgURE 3: The schematic of the proposed PAP method. The dotted circle is the area need to be padded with zero. The solid and dotted gray boxes, respectively, represent the vectors projected along the directions of red and green lines. The red and green blocks denote the results of the accumulation of pixel values along the red and green lines separately.

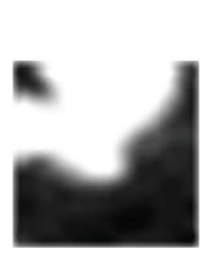

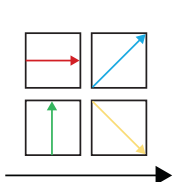

PAP
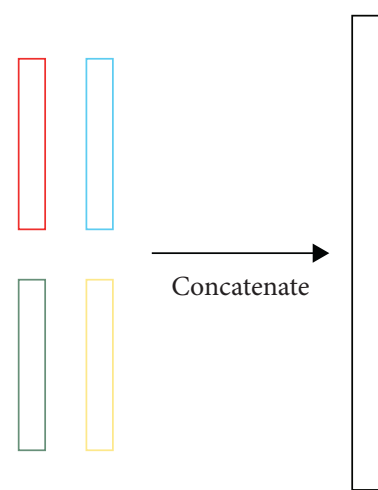

FIgURE 4: The details of the proposed PAP method. The red, blue, green, and yellow boxes represent the $1 \mathrm{D}$ vectors projected in the directions that angles with $x$-axis are $0,45,90$, and 135 degrees. The black box denotes the concatenated 1D vector.

size from $384 * 320$ to $768 * 672$. They are then normalized to be $512 * 512$. Among these samples, 21 datasets contain two or more intracranial aneurysms, and the remaining 145 cases only have one intracranial aneurysm. The number of intracranial aneurysms in all samples is 187 with the average diameter of $7.5 \mathrm{~mm}$. The minimum and maximum diameters are 2.4 and $23 \mathrm{~mm}$. Figure 9 shows the diameter's distribution. It can be found that most of them are between $5 \mathrm{~mm}$ and $10 \mathrm{~mm}$. Under the guidance of radiologists, 17 undistinguishable samples are removed and the remaining 170 available samples are taken as positive samples. Meanwhile, the 180 negative samples without intracranial aneurysms are tailored from the original volume.

3.2. Data Argumentation. To expand the training samples, we perform translation and rotation for the original volume. The MIP images after augmenting by the two strategies are shown in Figure 10. Compared with the translation-based method, more diverse samples can be obtained by rotating the volume. After rotating the volume with several angles, we tailor ROIs from the rotated volumes to obtain MIP images. The specific steps are as follows. (1) The original DICOM images are resampled after preprocessing to make the TOF MRA volume isotropic according to [32]. (2) The blood vessels are detected based on OTSU algorithm [26]. (3) Taking the center of the aneurysm as origin, the TOF MRA volumes are randomly rotated around $x-, y$ - and $z$-axis with plus or minus $0,5,10,15,20,25$, or 30 degrees to augment $40,30,20,10,5$, and 3 times. (4) A series of ROIs with size of $16 * 16 * 16$ are tailored from the rotated volumes. The augmented samples are divided into training, validation, and test sets according to the way of Table 4 .

3.3. Evaluation Metrics. Four metrics are usually used in evaluating the binary classification. TP indicates the proportion that the positive sample is correctly predicted as positive, FN means the ratio that the positive sample is incorrectly classified as negative, FP denotes proportion that the negative sample is wrongly predicted to be positive, and $\mathrm{TN}$ represents the ratio that the negative sample is true classified to be negative. In these experiments, the following four metrics are employed to quantitatively evaluate the performance of different methods on basis of above binary classification metrics. They are accuracy, precision, recall (also called sensitivity), and F1 score [33]. Accuracy denotes the proportion of the correct classification, precision refers to the correct proportion of positive samples, sensitivity indicates that the proportion of positive samples is classified as positive, and F1 score is the average between precision and sensitivity. These metrics could be computed by the following equations: 


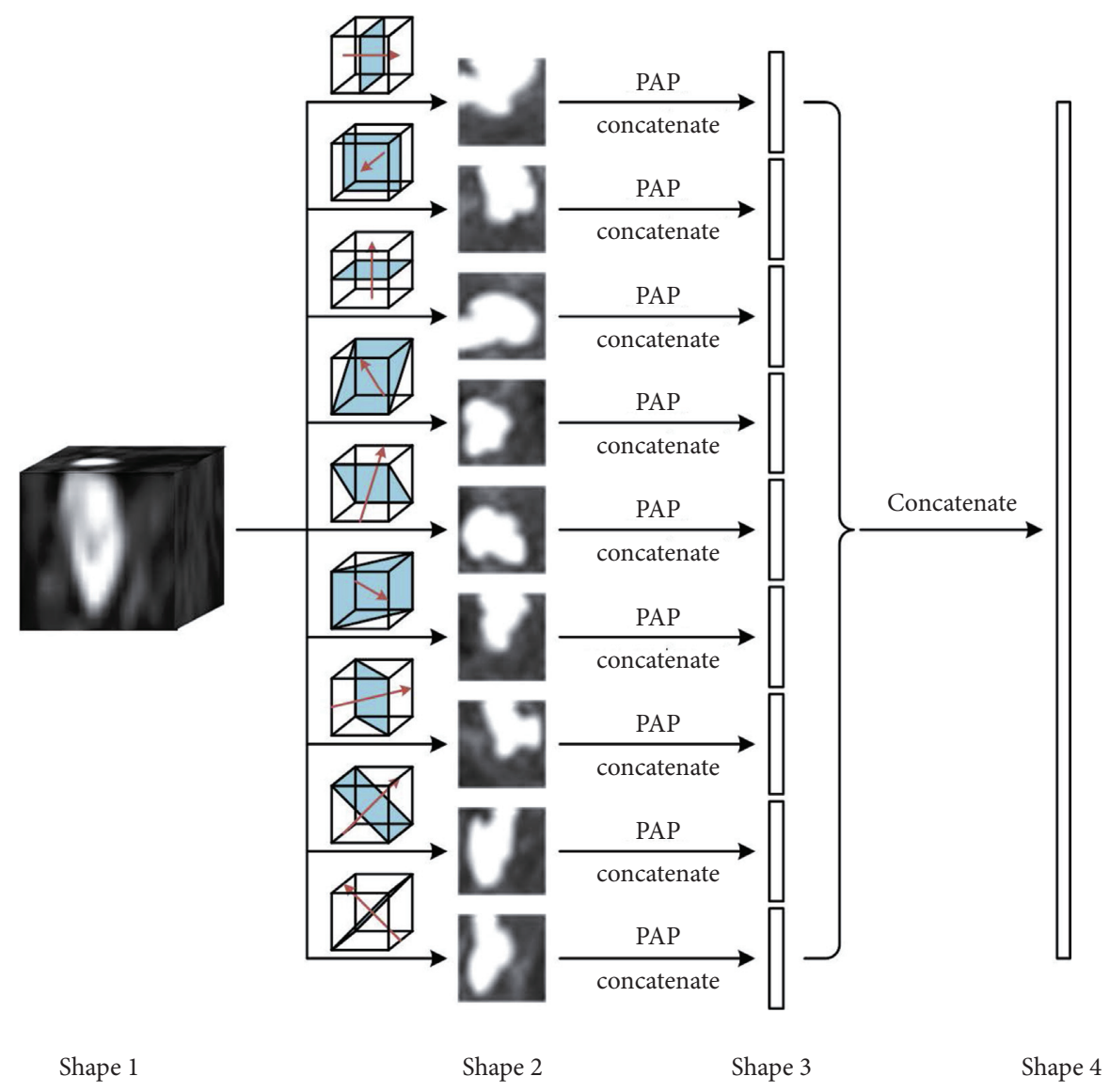

Figure 5: The generation of $1 \mathrm{D}$ vector. The black boxes denote the concatenated $1 \mathrm{D}$ vectors.

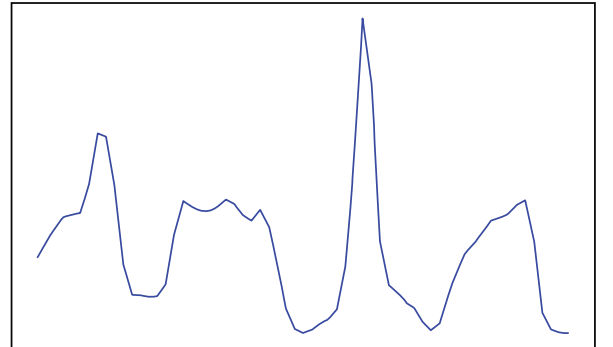

(a)

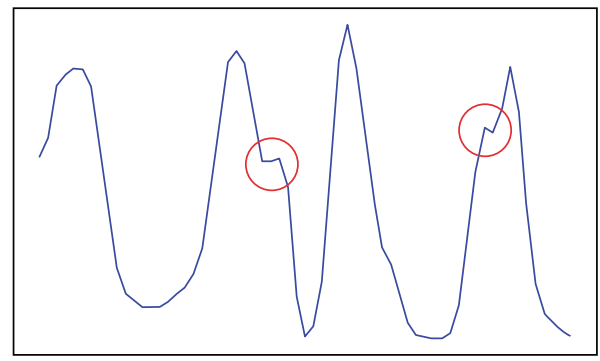

(c)

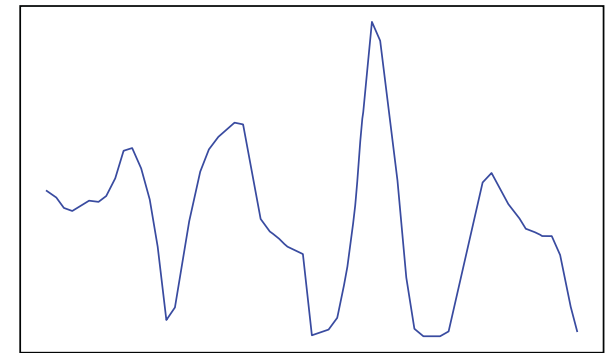

(b)

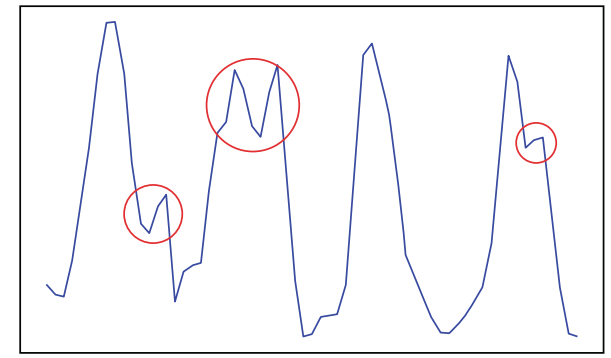

(d)

Figure 6: Examples of 1D samples. (a and b) denote the negative samples (arteries). (c and d) represent the positive samples (aneurysms). The red circles indicate the characteristics of the aneurysms. 
TABLe 1: The four kinds of data.

\begin{tabular}{cccc}
\hline Shape & Data & Size & Compression ratio \\
\hline shape $_{1}$ & 3D ROI & $16^{*} 16^{*} 16$ & - \\
shape $_{2}$ & 2D MIP image & $16^{*} 144$ & $56.25 \%$ \\
shape $_{3}$ & 1D vectors & $9^{*} 64$ & $14.06 \%$ \\
shape $_{4}$ & 1D convector & 576 & $14.06 \%$ \\
\hline
\end{tabular}

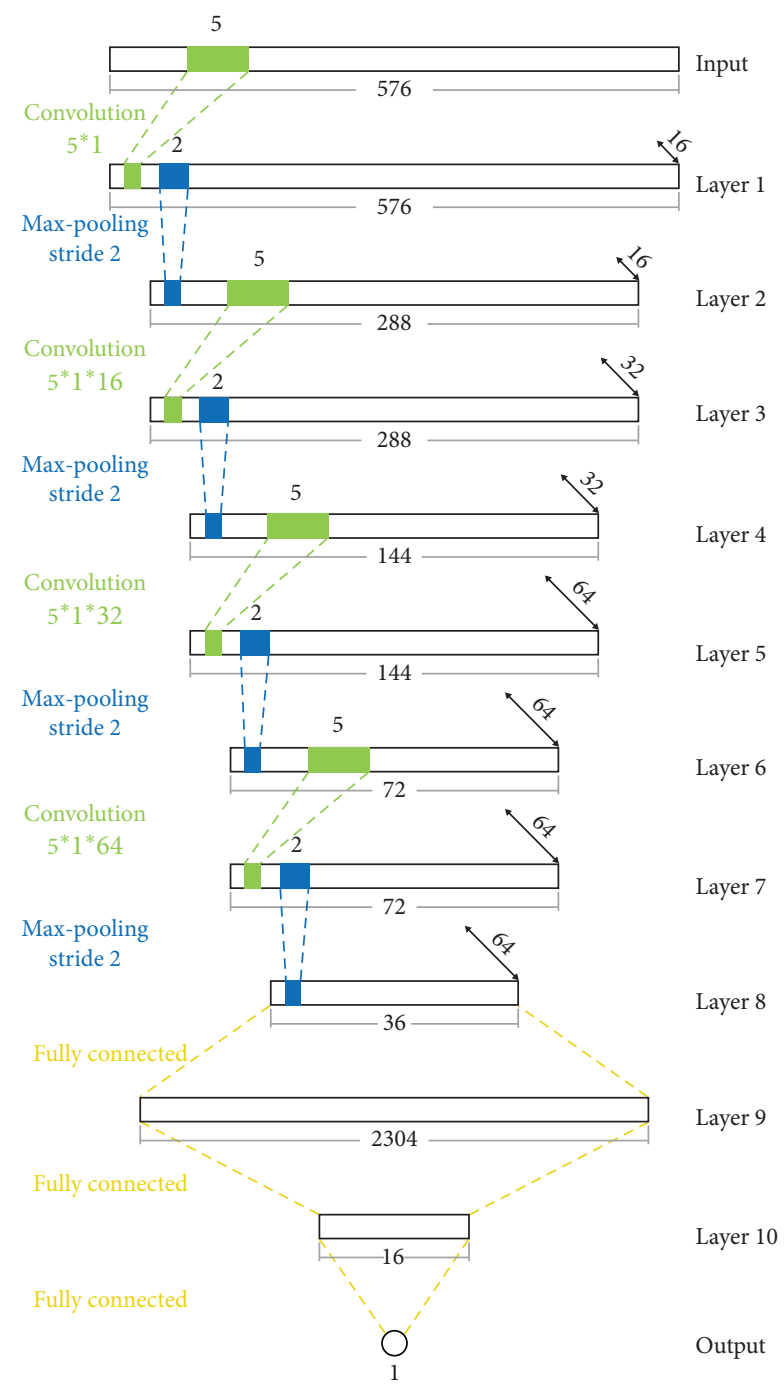

FIgURE 7: The hierarchical architecture of Model 1.

$$
\begin{aligned}
\text { Accuracy } & =\frac{\mathrm{TP}+\mathrm{TN}}{\mathrm{TP}+\mathrm{TN}+\mathrm{FP}+\mathrm{FN}}, \\
\text { Precision } & =\frac{\mathrm{TP}}{\mathrm{TP}+\mathrm{FP}}, \\
\text { Recall } & =\frac{\mathrm{TP}}{\mathrm{TP}+\mathrm{FN}}, \\
F_{1} & =\frac{2 \mathrm{TP}}{2 \mathrm{TP}+\mathrm{FP}+\mathrm{FN}} .
\end{aligned}
$$

In addition, Receiver Operating Characteristic (ROC) curves with Area Under the Curve (AUC) is also employed to evaluate the performance of different methods [34]. Taking the proportion of FP and sensitivity as the abscissa and ordinate, respectively, ROC can effectively express the case of TP and FP in different cases of thresholds. By calculating the area under the ROC curve, AUC can represent the portion that the predicted positive sample precedes the negative sample. It is noted that the closer that AUC is to 1, the better the result is. The value of AUC is computed as follows:

$$
\mathrm{AUC}=\frac{\sum_{i \in p} \operatorname{rank}_{i}(m *(m+1) / 2)}{m * n},
$$

where $\operatorname{rank}_{i}$ is taken to the $i$ th sample in a probabilistic order, $p$ represents the positive class, and $m$ and $n$ are the number of the positive and negative samples.

\section{Experiment Result}

The proposed method projects each MIP image to several 1D vectors by accumulating the pixels in several directions and then establishes a 1D CNN to detect intracranial aneurysms. It is to accelerate the training and reduce the demand for the samples. To validate the effectiveness of the proposed method, three kinds of experiments are conducted.

4.1. Training with Different Number of $1 D$ Vectors. It is clear that the accuracy of aneurysm detection is lower in case of fewer projections (1D vectors). However, fewer $1 \mathrm{D}$ vectors means that the training can be highly accelerated. To trade off the accuracy and efficiency, an appropriate number of projections will be investigated. Specifically, three groups of $1 \mathrm{D}$ vectors are generated by projecting MIP image along 2,4 , and 8 directions, and they are divided into training, validation, and test sets. It is worth noting that two kinds of samples are generated for each case, which correspond to shape $_{3}$ and shape ${ }_{4}$ listed in Table 1 . Then, 9800 samples (4760 positive samples and 5040 negative samples) are taken to train two models shown in Figures 7 and 8 with GPU. After training, we test on 2800 test samples (1360 positive samples and 1440 negative samples). Their results are shown in Table 5. The ROC curves under different projection configurations are illustrated as Figures 11 and 12. It can be found that the performance and computation of two models both increase in case of more inputs. That is, the performance of 4 projections is significantly better than the case of 2. Compared with the latter, the accuracy and precision are raised by about 2 and 3\%. Yet, their computation amounts are close. However, the performances will not increase obviously while the projection number reaches 8 . The possible reason is that more projections could not detect more features from the MIP image, but it causes more computation. Considering them, the 4 projections are taken in the following experiments.

4.2. Training with Different Number of Samples. To verify the effectiveness and robustness of the proposed method, the above two 1D networks are utilized to conduct two 


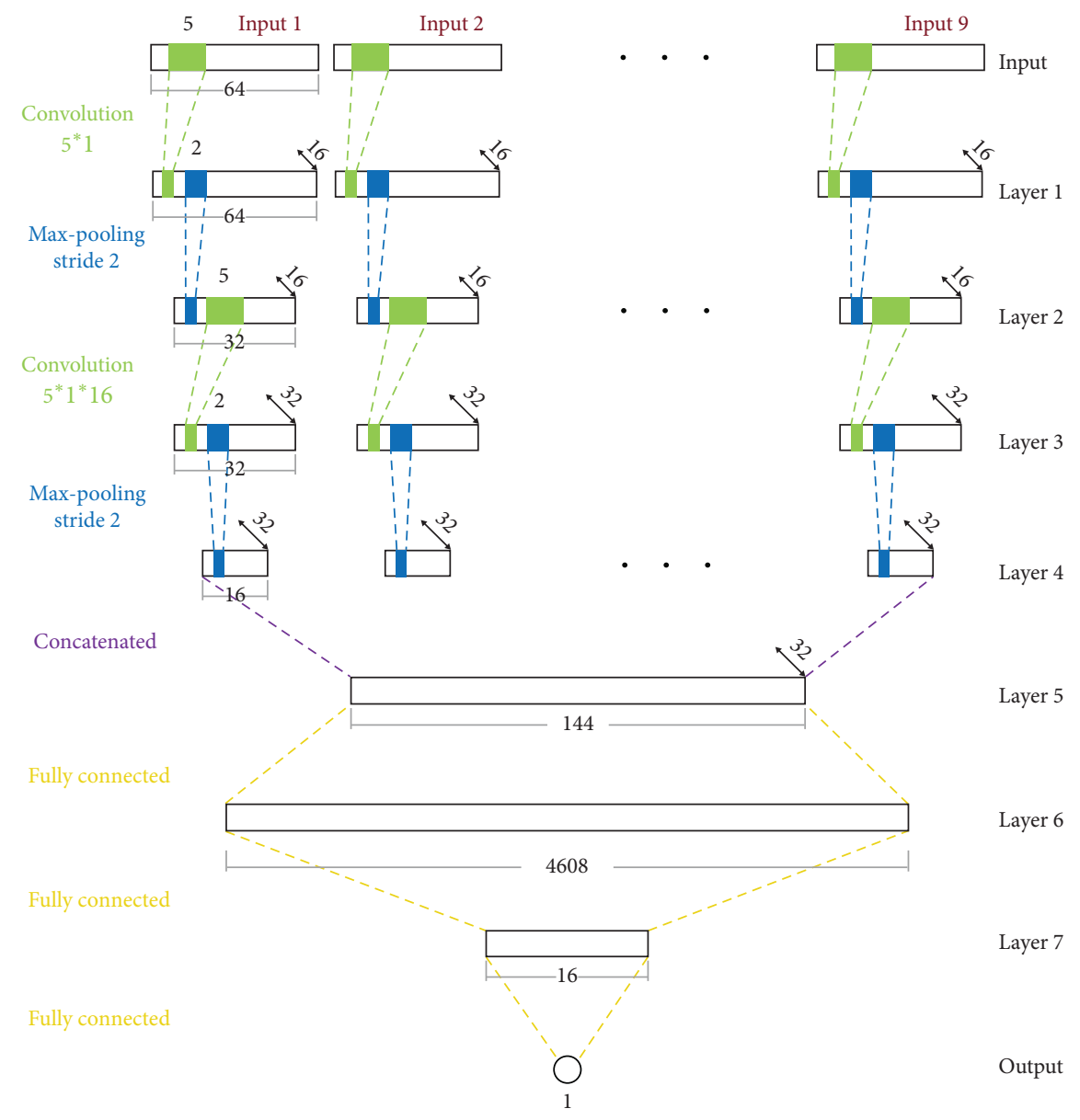

Figure 8: The hierarchical architecture of Model 2.

TABLE 2: The architectures of 1D CNN discrimination Model 1.

\begin{tabular}{lcccc}
\hline Layers & Feature vector & Kernel size & Stride & Output size \\
\hline Input & 1 & - & - & 576 \\
Convolution & 16 & 5 & 1 & 576 \\
Max pooling & 16 & 2 & 2 & 288 \\
Convolution & 32 & 5 & 1 & 288 \\
Max pooling & 32 & 2 & 2 & 144 \\
Convolution & 64 & 5 & 1 & 144 \\
Max pooling & 64 & 2 & 2 & 72 \\
Convolution & 64 & 5 & 1 & 72 \\
Max pooling & 64 & 2 & 2 & 36 \\
Fully connected & 2304 & - & - & 2304 \\
Fully connected & 16 & - & - & 16 \\
Output & 1 & - & - & 1 \\
\hline
\end{tabular}

comparative experiments with the same original samples in cases of different argumentations. Meanwhile, two corresponding 2D CNNs with the similar architectures are also trained using the corresponding MIP images. These training tasks are conducted using GPU and CPU. The results are shown in Tables 6 and 7. The more the samples are, the better the results are for all cases. When training data becomes less (data ${ }_{1}$, data $_{2}$, and data 3 listed in Table 4 ), the performance of the 1D CNNs are better than that of 2D CNN. The accuracy of $1 \mathrm{D} \mathrm{CNN}$ is about $1 \%$ to $4 \%$ higher than the latter, and the
TABLE 3: The architectures of 1D CNN discrimination Model 2.

\begin{tabular}{lcccc}
\hline Layers & Feature vector & Kernel size & Stride & Output size \\
\hline Input & 1 & - & - & $64^{*} 9$ \\
Convolution & 16 & 5 & 1 & $64^{*} 9$ \\
Max pooling & 16 & 2 & 2 & $32^{*} 9$ \\
Convolution & 32 & 5 & 1 & $32^{*} 9$ \\
Max pooling & 32 & 2 & 2 & $16^{*} 9$ \\
Concatenated & 32 & - & - & 144 \\
Fully connected & 4608 & - & - & 4608 \\
Fully connected & 16 & - & - & 16 \\
Output & 1 & - & - & 1
\end{tabular}

precision and sensitivity are also slightly higher. It can be found that the $2 \mathrm{D} \mathrm{CNN}$ is more sensitive to the number of samples. That is, there is no significant difference among these models, especially when a large number of training samples such as data 6 and data 7 are utilized. In the case of training with GPU, the cost of $1 \mathrm{D}$ CNNs of each epoch is less than these of 2DCNNs. Moreover, the GPU utilization rate for $1 \mathrm{D} C N N$ is about $1 / 3$ to $2 / 5$ of that of $2 \mathrm{D} C N N$. When training with $\mathrm{CPU}$, the CPU is almost $100 \%$ employed and the time in training $1 \mathrm{D} C N N$ are much less than that of MIP image-based case. It is because that $1 \mathrm{D}$ CNN effectively reduces the size of data and then simplifies the network. 


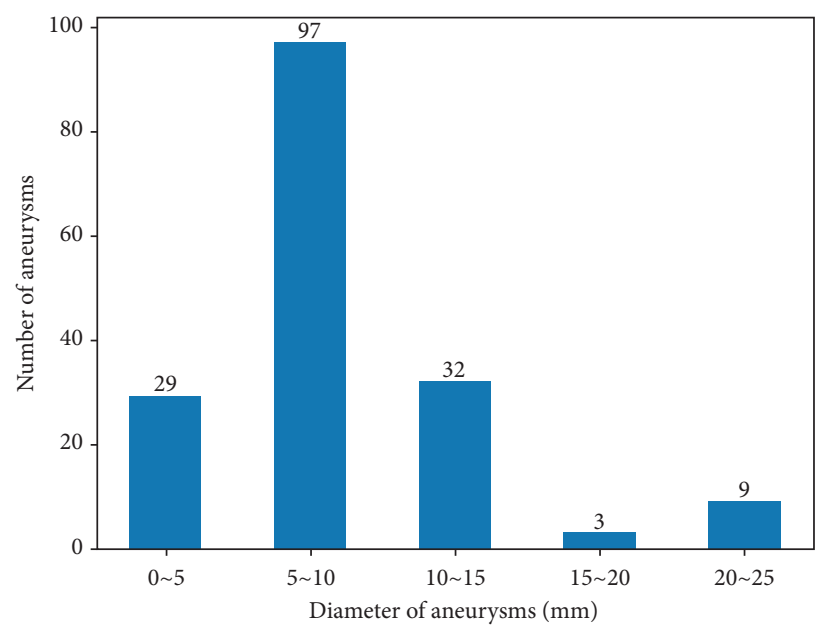

Figure 9: The distribution of aneurysm diameter.

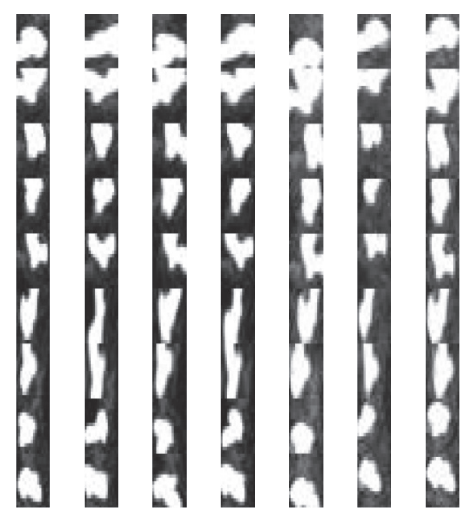

$\begin{array}{llllll}\text { (a) } & \text { (b) } & \text { (c) } & \text { (d) } & \text { (e) } & \text { (f) }\end{array}$

FIgURE 10: Examples of rotation and translation: (a) the original MIP image, (b-d) the rotational samples, and (e-g) the translational samples.

TABLE 4: The details of the dataset.

\begin{tabular}{lcccccc}
\hline \multirow{2}{*}{ Dataset } & \multicolumn{2}{c}{ Training } & \multicolumn{2}{c}{ Validation } & \multicolumn{2}{c}{ Test } \\
& Aneurysm & Artery & Aneurysm & Artery & Aneurysm & Artery \\
\hline Data1 (original) & 126 & 119 & 18 & 17 & 36 & 34 \\
Data2 (3 times) & 378 & 357 & 54 & 51 & 108 & 102 \\
Data3 (5 times) & 630 & 595 & 90 & 1850 & 1050 \\
Data4 (10 times) & 1260 & 1190 & 180 & 170 & 360 & 180 \\
Data5 (20 times) & 2520 & 2380 & 360 & 340 & 720 & 340 \\
Data6 (30 times) & 3780 & 3570 & 540 & 510 & 1080 & 680 \\
Data7 (40 times) & 5040 & 4760 & 720 & 680 & 1440 & 1020 \\
\hline
\end{tabular}

TABLE 5: Results of different projection number configurations.

\begin{tabular}{ccccccccc}
\hline 1D CNN & Number & Input & ACC (\%) & PRE (\%) & SEN (\%) & AUC & Ratio (\%) & Time (s) \\
\hline \multirow{3}{*}{ Model 1 } & 2 & $288^{*} 1$ & 93.28 & 92.34 & 93.97 & 0.9811 & 24 & 1.6476 \\
& 4 & $576^{*} 1$ & 95.21 & 96.87 & 93.17 & 0.9861 & 27 & 2.1206 \\
& 8 & $1152^{*} 1$ & 95.11 & 96.36 & 93.45 & 0.9874 & 29 \\
Model 2 & 2 & $9^{*} 32^{*} 1$ & 93.86 & 93.55 & 93.82 & 0.9832 & 10 & 3.5536 \\
\hline & 4 & $9^{*} 64^{*} 1$ & 95.32 & 96.03 & 94.26 & 0.9882 & 14 & 3.2719 \\
& 8 & $9^{*} 128^{*} 1$ & 95.86 & 96.21 & 95.22 & 0.9912 & 18 & 3.8915 \\
\hline
\end{tabular}




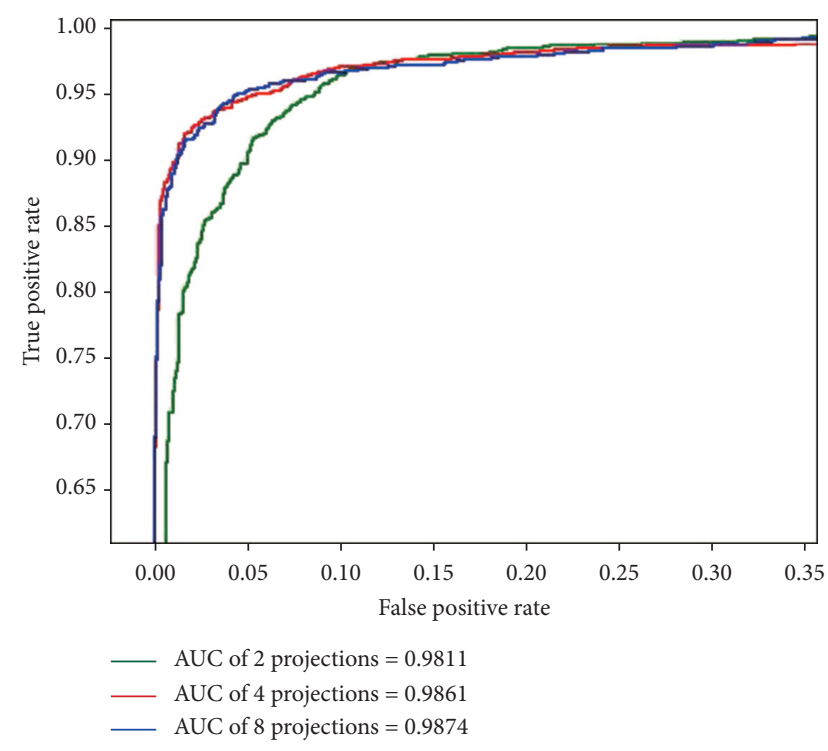

FIGURE 11: ROC curves of Model 1 with different projection number configurations.

These experiments show that the proposed method is effective and robust especially when the samples are rare.

4.3. Compared with Other Methods. This section will compare our two 1D CNNs with two classic models [20,21]. The first model is composed of two convolution layers, two maximum pooling layers, and three two-connected layers. Its input is the original ROI sample as the shape ${ }_{1}$ and the output is the probability whether an aneurysm is located in this ROI. The second one employs the concatenated MIP images to detect aneurysms on basis of 2D CNN. 9 MIP images are vertically concatenated as the input of the network, which consists of two convolution layers, two maximum pooling layers, and two fully connected layers. Our first model is shown in Figure 7. It is an 11-layer 1D CNN with four convolution layers, four maximum pooling layers, and two fully connected layers. The input of this model is a $5761 \mathrm{D}$ vector as the shape $_{4}$. The last one employs a multichannel network model with the input of nine $641 \mathrm{D}$ vectors ( shape $_{3}$ ). For each channel, a 4-layer CNN composed by two convolution layers and two maximum pooling layers is utilized to extract aneurysm features. After concatenating these features, two fully connected layers are used to do classification.

After training the four models with 9800 samples on GPU, the results are listed in Table 8. The ROC curves of these methods are denoted in Figure 13. The method of ours (con-input) means the result of the third model is trained with the concatenated $1 \mathrm{D}$ vector $\left(\right.$ shape $_{4}$ in Table 1 ), while ours (9 inputs) denotes that of the last model trained by nine $641 \mathrm{D}$ vectors ( shape $_{3}$ in Table 1 ). It is clear that the performances of our proposed method are similar to [20], which are slightly better than that in [21]. The model with multichannel outperforms other three strategies, especially in terms of sensitivity. In addition, the calculation of our method becomes easy in case of GPU training.

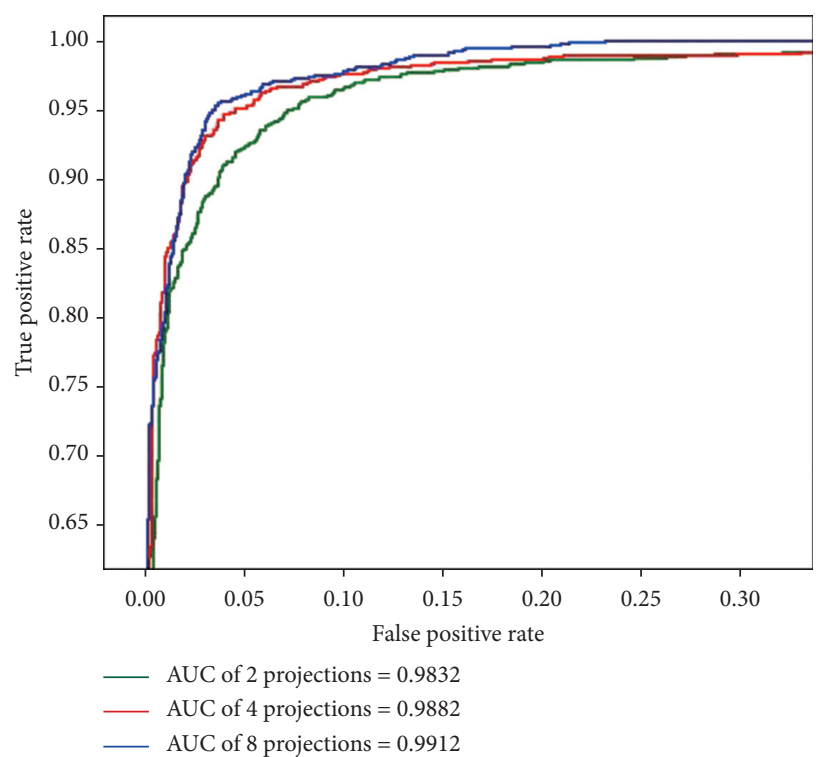

FIgURE 12: ROC curves of Model 2 with different projection number configurations.

\section{Discussion}

There are many research studies about intracranial aneurysm detection and classification on the basis of the $2 \mathrm{D}$ or $3 \mathrm{D}$ CNN $[18,19,21,24]$. To the best of our knowledge, however, this is the first time that $1 \mathrm{D}$ CNN based is used in this application. Inspired by the MIP, the $1 \mathrm{D}$ vectors are generated by accumulating pixels of the 2D MIP image in certain directions. Similar to the principle of CT imaging, these $1 \mathrm{D}$ vectors contain the main features of the related MIP images. What is important is that $1 \mathrm{D}$ CNN replaces the traditional 2D CNN, which greatly reduces the number of parameters and simplifies the training complexity. Meanwhile, we compare the proposed strategy with the traditional 2D CNN-based methods. It has been proved that the results of our method are close to or even better than those based on 2D CNN. Moreover, our method can conduct the training with fewer samples on the CPU platform. In contrast, 2D $\mathrm{CNN}$ training requires more training samples and highperformance GPU. That is, the proposed method reduces the demands for samples and computer performance. In addition, by comparing our proposed strategy with the existing traditional methods, it is clear that our method performs equally well. Obviously, the proposed strategy can be extent to other tasks including the feature detection and image classification. In our experiment, all the original data are augmented by $3 \mathrm{D}$ rotation. Traditional two-dimensional translation and rotation are common methods of data expansion. However, limited by the rotation angle and translation distance, the effect of these methods is not ideal for our small data. The augmented images show a strong correlation with the original image. In our application, 3D rotation is applied to increase the original data, which makes the augmented data more diverse. Obviously, the 3D rotation method can be applied for more data augmentation processes. 
TABLE 6: Detection results of Model 1 under different samples.

\begin{tabular}{|c|c|c|c|c|c|c|c|c|c|c|}
\hline \multirow{2}{*}{ Data } & \multirow{2}{*}{$\mathrm{TR}$} & \multirow{2}{*}{ VAL } & \multirow{2}{*}{$\mathrm{TE}$} & \multirow{2}{*}{ Model } & \multicolumn{3}{|c|}{ Performance (\%) } & \multicolumn{2}{|c|}{ GPU } & \multirow{2}{*}{$\mathrm{CPU}(\min )$} \\
\hline & & & & & $\mathrm{ACC}$ & PRE & SEN & Ratio (\%) & Time (s) & \\
\hline \multirow{2}{*}{ Data $_{1}$} & \multirow{2}{*}{245} & \multirow{2}{*}{35} & \multirow{2}{*}{70} & $1 \mathrm{D}$ & 88.57 & 90.63 & 85.29 & 19 & 0.13 & 0.45 \\
\hline & & & & $2 \mathrm{D}$ & 84.28 & 82.35 & 84.85 & 48 & 0.16 & 3.76 \\
\hline \multirow[b]{2}{*}{ Data $_{2}$} & \multirow{2}{*}{735} & \multirow{2}{*}{105} & \multirow{2}{*}{210} & $1 \mathrm{D}$ & 90.00 & 90.09 & 89.22 & 23 & 0.26 & 1.36 \\
\hline & & & & $2 \mathrm{D}$ & 87.62 & 85.85 & 89.22 & 54 & 0.34 & 11.00 \\
\hline \multirow{2}{*}{ Data $_{3}$} & \multirow{2}{*}{1225} & \multirow{2}{*}{175} & \multirow{2}{*}{350} & $1 \mathrm{D}$ & 90.28 & 88.64 & 91.76 & 24 & 0.40 & 2.26 \\
\hline & & & & $2 \mathrm{D}$ & 89.71 & 88.95 & 90.00 & 56 & 0.51 & 16.44 \\
\hline \multirow{2}{*}{ Data $_{4}$} & \multirow{2}{*}{2450} & \multirow{2}{*}{350} & \multirow{2}{*}{700} & $1 \mathrm{D}$ & 93.86 & 93.55 & 93.82 & 26 & 0.70 & 4.50 \\
\hline & & & & $2 \mathrm{D}$ & 93.57 & 92.75 & 94.12 & 58 & 0.78 & 32.86 \\
\hline \multirow{2}{*}{ Data $_{5}$} & \multirow{2}{*}{4900} & \multirow{2}{*}{700} & \multirow{2}{*}{1400} & $1 \mathrm{D}$ & 94.64 & 96.04 & 92.79 & 27 & 1.21 & 8.95 \\
\hline & & & & $2 \mathrm{D}$ & 93.93 & 93.94 & 93.53 & 60 & 1.76 & 66.70 \\
\hline \multirow{2}{*}{ Data $_{6}$} & \multirow{2}{*}{7350} & \multirow{2}{*}{1050} & \multirow{2}{*}{2100} & $1 \mathrm{D}$ & 94.90 & 95.97 & 93.43 & 27 & 1.83 & 13.43 \\
\hline & & & & $2 \mathrm{D}$ & 94.62 & 94.95 & 93.92 & 61 & 2.61 & 102.50 \\
\hline \multirow{2}{*}{ Data $_{7}$} & \multirow{2}{*}{9800} & \multirow{2}{*}{1400} & \multirow{2}{*}{2800} & $1 \mathrm{D}$ & 95.21 & 96.87 & 93.16 & 27 & 2.12 & 17.86 \\
\hline & & & & $2 \mathrm{D}$ & 94.85 & 95.57 & 96.51 & 62 & 3.44 & 136.79 \\
\hline
\end{tabular}

TABLE 7: Detection results of Model 2 under different samples.

\begin{tabular}{|c|c|c|c|c|c|c|c|c|c|c|}
\hline \multirow{2}{*}{ Data } & \multirow{2}{*}{$\mathrm{TR}$} & \multirow{2}{*}{ VAL } & \multirow{2}{*}{$\mathrm{TE}$} & \multirow{2}{*}{ Model } & \multicolumn{3}{|c|}{ Performance (\%) } & \multicolumn{2}{|c|}{ GPU } & \multirow{2}{*}{$\mathrm{CPU}(\min )$} \\
\hline & & & & & $\mathrm{ACC}$ & PRE & SEN & Ratio (\%) & Time (s) & \\
\hline \multirow[b]{2}{*}{ Data $_{1}$} & \multirow[b]{2}{*}{245} & \multirow{2}{*}{35} & \multirow{2}{*}{70} & $1 \mathrm{D}$ & 90.00 & 88.57 & 91.18 & 8 & 0.24 & 0.01 \\
\hline & & & & $2 \mathrm{D}$ & 88.57 & 88.24 & 88.24 & 25 & 0.26 & 1.39 \\
\hline \multirow{2}{*}{ Data $_{2}$} & \multirow{2}{*}{735} & \multirow{2}{*}{105} & \multirow{2}{*}{210} & $1 \mathrm{D}$ & 90.48 & 91.84 & 88.24 & 9 & 0.39 & 0.02 \\
\hline & & & & $2 \mathrm{D}$ & 89.05 & 90.72 & 86.27 & 28 & 0.37 & 4.07 \\
\hline \multirow{2}{*}{ Data $_{3}$} & \multirow{2}{*}{1225} & \multirow{2}{*}{175} & \multirow{2}{*}{350} & $1 \mathrm{D}$ & 91.71 & 92.22 & 90.59 & 10 & 0.57 & 0.02 \\
\hline & & & & $2 \mathrm{D}$ & 90.86 & 91.57 & 89.41 & 32 & 0.51 & 6.82 \\
\hline \multirow{2}{*}{ Data $_{4}$} & \multirow{2}{*}{2450} & \multirow{2}{*}{350} & \multirow{2}{*}{700} & $1 \mathrm{D}$ & 93.26 & 93.73 & 92.35 & 12 & 0.92 & 0.03 \\
\hline & & & & $2 \mathrm{D}$ & 92.71 & 93.66 & 91.18 & 36 & 0.84 & 13.75 \\
\hline \multirow{2}{*}{ Data $_{5}$} & \multirow{2}{*}{4900} & \multirow{2}{*}{700} & \multirow{2}{*}{1400} & $1 \mathrm{D}$ & 93.86 & 94.46 & 92.79 & 13 & 1.65 & 0.05 \\
\hline & & & & $2 \mathrm{D}$ & 92.86 & 93.94 & 91.18 & 39 & 1.58 & 27.03 \\
\hline \multirow{2}{*}{ Data $_{6}$} & \multirow{2}{*}{7350} & \multirow{2}{*}{1050} & \multirow{2}{*}{2100} & $1 \mathrm{D}$ & 94.43 & 95.20 & 93.24 & 14 & 2.46 & 0.07 \\
\hline & & & & $2 \mathrm{D}$ & 94.10 & 95.07 & 92.65 & 41 & 2.37 & 40.30 \\
\hline \multirow{2}{*}{ Data $_{7}$} & \multirow{2}{*}{9800} & \multirow{2}{*}{1400} & \multirow{2}{*}{2800} & $1 \mathrm{D}$ & 95.32 & 96.03 & 94.26 & 14 & 3.27 & 0.10 \\
\hline & & & & $2 \mathrm{D}$ & 94.46 & 94.80 & 93.75 & 41 & 3.08 & 53.74 \\
\hline
\end{tabular}

TABle 8: Comparison with other methods.

\begin{tabular}{|c|c|c|c|c|c|c|c|c|}
\hline Methods & Input & ACC (\%) & PRE (\%) & SEN (\%) & AUC & F1 & Ratio (\%) & Time (s) \\
\hline Dou & $16^{*} 16^{*} 16$ & 95.25 & 95.82 & 94.34 & 0.9870 & 95.07 & 43 & 3.9375 \\
\hline Nakao & $16^{*} 144$ & 94.07 & 94.09 & 93.68 & 0.9895 & 93.88 & 55 & 1.8983 \\
\hline Ours (con-input) & $576^{*} 1$ & 95.21 & 96.87 & 93.16 & 0.9861 & 94.98 & 27 & 2.1206 \\
\hline Ours (9 inputs) & $9 * 64^{*} 1$ & 95.86 & 96.21 & 95.22 & 0.9912 & 95.71 & 15 & 3.8915 \\
\hline
\end{tabular}

It should be admitted that the data lost information in the process of dimensionality reduction. On the contrary, the removal of redundant information makes the detection and classification easier. In the proposed method, the number of $1 \mathrm{D}$ vectors has been demonstrated to be a key factor in the controlled experiments. The more the $1 \mathrm{D}$ vectors are, the more feature information of the data will be retained and the classification result will be better. Yet, more $1 \mathrm{D}$ vectors will increase the training computation. Then, a balance should be determined in specific applications. 


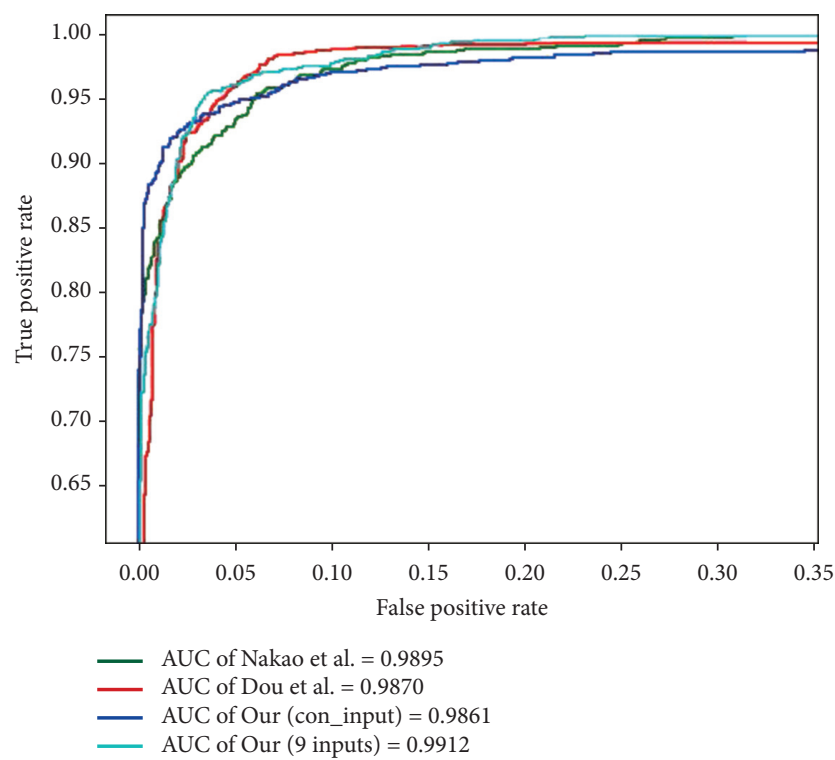

Figure 13: ROC curves of different methods.

\section{Conclusion}

This paper proposes an IAs detection by introducing the $1 \mathrm{D} \mathrm{CNN}$ to do classification. Inspired by the MIP-based IAs detection method, we further generate several $1 \mathrm{D}$ vectors from each MIP image and then input the $1 \mathrm{D}$ vectors to the $1 \mathrm{D} C \mathrm{CNN}$ to determine whether there is an IA or not in the considered 3D image patch, i.e., the MIP strategy-based method transfers the traditional 3D intracranial aneurysms detection into 2D image classification problem and we then transfer it into a $1 \mathrm{D}$ case. The size of the one-dimensional vector generated by our method is $14.06 \%$ of the original $3 \mathrm{D}$ data and $25 \%$ of the 2D MIP image. Correspondingly, the parameter of a 1D network is $44.23 \%$ of that of a $2 \mathrm{D}$ network. As such, this way greatly accelerates the training process and reduces the demands for samples in training the CNN. According to the experiments, our strategy is simple and effective. It should be mentioned that the proposed strategy achieves 95.86\% detection accuracy, which is even better than the traditional methods. The efficiency of the proposed method outperforms the classic MIP about 10 times in CPU training. This makes it possible to train with CPU in the clinical settings. In addition, this strategy can be used to other high 2D- and 3D-image-related applications which may greatly reduce the difficulty in training. Meanwhile, there are other issues to deserve further attention. The first is that how to generate the $1 \mathrm{D}$ vector from the MIP image more efficiently. This paper simply uses the way of accumulating all pixels' intensities along each direction. This is just an example. Other sophisticated ways need to be researched. The second is that $1 \mathrm{D}$ CNN structure and optimization should be analyzed to further improve the classification accuracy. Future work should involve clinical data acquisition, preprocessing, etc.

\section{Data Availability}

The data in the manuscript are patient related. The data include some private information. The researchers who are interested in the data can contact the corresponding author through the email.

\section{Conflicts of Interest}

The authors declare that they have no conflicts of interest.

\section{Acknowledgments}

This study was supported by the National Key Research and Development Program of China (Grant no. 2017YFBI1303100).

\section{References}

[1] X. Yang, D. J. Blezek, L. T. E. Cheng, W. J. Ryan, D. F. Kallmes, and B. J. Erickson, "Computer-aided detection of intracranial aneurysms in MR angiography," Journal of Digital Imaging, vol. 24, no. 1, pp. 86-95, 2011.

[2] M. H. Vlak, A. Algra, R. Brandenburg, and G. J. Rinkel, "Prevalence of unruptured intracranial aneurysms, with emphasis on sex, age, comorbidity, country, and time period: a systematic review and meta-analysis," The Lancet Neurology, vol. 10, no. 7, pp. 626-636, 2011.

[3] T. Abboud, J. Rustom, M. Bester et al., "Morphology of ruptured and unruptured intracranial aneurysms," World Neurosurgery, vol. 99, pp. 610-617, 2017.

[4] M. Herzberg, R. Forbrig, C. Schichor, H. Brückmann, and F. Dorn, "Preoperative digital subtraction angiography in incidental unruptured intracranial aneurysms," Clinical Neuroradiology, vol. 28, no. 3, pp. 429-435, 2018.

[5] A. Leffers and A. Wagner, "Neurologic complications of cerebral angiography: a retrospective study of complication rate and patient risk factors," Acta Radiologica, vol. 41, no. 3 , pp. 204-210, 2000.

[6] O. Rustemi, A. Alaraj, S. Shakur et al., "Detection of unruptured intracranial aneurysms on noninvasive imaging. is there still a role for digital subtraction angiography?" Surgical Neurology International, vol. 6, no. 1, p. 175, 2015.

[7] M. Cirillo, F. Scomazzoni, L. Cirillo, and M. Cadioli, "Comparison of 3D TOF-MRA and 3D CE-MRA at 3T for imaging of intracranial aneurysms," European Journal of Radiology, vol. 82, pp. e853-e859, 2013.

[8] A. Faron, T. Sichtermann, N. Teichert et al., "Performance of a deep-learning neural network to detect intracranial aneurysms from 3D TOF-MRA compared to human readers," Clinical Neuroradiology, vol. 30, no. 3, pp. 591-598, 2019.

[9] Y. Kwak, W. Son, Y.-S. Kim, J. Park, and D.-H. Kang, "Discrepancy between MRA and DSA in identifying the shape of small intracranial aneurysms," Journal of Neurosurgery, vol. 24, pp. 1-7, 2020.

[10] S. Miki, N. Hayashi, Y. Masutani et al., "Computer-assisted detection of cerebral aneurysms in $\mathrm{MR}$ angiography in a routine image-reading environment: effects on diagnosis by radiologists," American Journal of Neuroradiology, vol. 37, no. 6, pp. 1038-1043, 2016.

[11] S. Buksakowska, J. M. Accurso, F. Diehn et al., "Computeraided diagnosis improves detection of small intracranial aneurysms on MRA in a clinical setting," American Journal of Neuroradioology, vol. 35, no. 10, pp. 1897-1902, 2014. 
[12] Y. Wu, Y. Ma, J. Liu, J. Du, and L. Xing, "Self-attention convolutional neural network for improved MR image reconstruction," Information Sciences, vol. 490, pp. 317-328, 2019.

[13] Q. Dou, H. Chen, L. Yu, J. Qin, and P.-A. Heng, "Multilevel contextual 3-D CNNs for false positive reduction in pulmonary nodule detection," IEEE Transactions on Biomedical Engineering, vol. 64, no. 7, pp. 1558-1567, 2017.

[14] A. Krizhevsky, I. Sutskever, and G. E. Hinton, "ImageNet classification with deep convolutional neural networks," Communications of the ACM, vol. 60, no. 6, pp. 84-90, 2017.

[15] M. Havaei, A. Davy, D. Warde-Farley et al., "Brain tumor segmentation with deep neural networks," Medical Image Analysis, vol. 35, pp. 18-31, 2017.

[16] H. C. Kim, J. K. Rhim, J. H. Ahn, and J. J. Park, "Machine learning application for rupture risk assessment in small-sized intracranial aneurysm," Journal of Clinical Medicine, vol. 8, no. 5, 2019.

[17] T. Sichtermann, A. Faron, R. Sijben, N. Teichert, J. Freiherr, and M. Wiesmann, "Deep learning-based detection of intracranial aneurysms in 3D TOF-MRA," American Journal of Neuroradiology, vol. 40, no. 1, pp. 25-32, 2019.

[18] A. Park, C. Chute, P. Rajpurkar et al., "Deep learning-assisted diagnosis of cerebral aneurysms using the HeadXNet model," JAMA Network Open, vol. 2, no. 6, 2019.

[19] B. Joo, S. S. Ahn, P. H. Yoon et al., “A deep learning algorithm may automate intracranial aneurysm detection on MR angiography with high diagnostic performance," European Radiology, vol. 30, no. 11, pp. 5785-5793.

[20] Q. Dou, H. Chen, L. Yu et al., "Automatic detection of cerebral microbleeds from MR images via 3D convolutional neural networks," IEEE Transactions on Medical Imaging, vol. 35, no. 5, pp. 1182-1195, 2016.

[21] T. Nakao, S. Hanaoka, Y. Nomura et al., "Deep neural network-based computer-assisted detection of cerebral aneurysms in MR angiography," Journal of Magnetic Resonance Imaging, vol. 47, no. 4, pp. 948-953, 2018.

[22] J. N. Stember, P. Chang, D. M. Stember et al., "Convolutional neural networks for the detection and measurement of cerebral aneurysms on magnetic resonance angiography," Journal of Digital Imaging, vol. 32, no. 5, pp. 808-815, 2019.

[23] H. Duan, Y. Huang, L. Liu, H. Dai, L. Chen, and L. Zhou, "Automatic detection on intracranial aneurysm from digital subtraction angiography with cascade convolutional neural networks," BioMedical Engineering OnLine, vol. 18, no. 1, 2019.

[24] D. Ueda, A. Yamamoto, M. Nishimori et al., "Deep learning for MR angiography: automated detection of cerebral aneurysms," Radiology, vol. 290, no. 1, pp. 187-194, 2019.

[25] C.-C. Lin, M.-H. Sheu, H.-K. Chiang, C. Liaw, and Z.-C. Wu, "The efficient VLSI design of BI-CUBIC convolution interpolation for digital image processing," in Proceedings of the 2008 IEEE International Symposium on Circuits and Systems (ISCAS), vol. 1-10, p. 480, Seattle, WA, USA, May 2008.

[26] N. Otsu, "A threshold selection method from gray-level histograms," IEEE Transactions on System, Man and Cybernetics, vol. 9, no. 1, pp. 63-68, 1979.

[27] T. Koller, G. Gerig, G. Szekely, and D. Dettwiler, "Multiscale detection of curvilinear structures in 2-D and 3-D image data," in Proceedings of the IEEE International Conference on Computer Vision, pp. 864-869, Cambridge, MA, USA, June 1995.
[28] Z. Vrselja, H. Brkic, S. Mrdenovic, R. Radic, and G. Curic, "Function of circle of Willis," Journal of Cerebral Blood Flow \& Metabolism, vol. 34, no. 4, pp. 578-584, 2014.

[29] S. Ioffe and C. Szegedy, "Batch normalization: accelerating deep network training by reducing internal covariate shift," in Proceedings of the 32nd International Conference on Machine Learning, vol. 1, pp. 448-456, Lile, France, July 2015.

[30] V. Nair and G. E. Hinton, "Rectified linear units improve restricted Boltzmann machines," in Proceedings of the 27th International Conference on Machine Learning, pp. 807-814, Haifa, Israel, June 2010.

[31] K. He, X. Zhang, S. Ren, and J. Sun, "Delving deep into rectifiers: surpassing human-level performance on ImageNet classification," in Proceedings of the 2015 IEEE International Conference on Computer Vision, pp. 1026-1034, Santiago, Chile, December 2015.

[32] L. Nyúl and J. Udupa, "On standardizing the MR image intensity scale," Magnetic Resonance in Medicine, vol. 42, no. 6, pp. 1072-1081, 1999.

[33] D. M. W. Powers, "Evaluation: from precision, recall and Fmeasure to roc., informedness, markedness \& correlation," Journal of Machine Learning Technologies, vol. 2, pp. 37-63, 2011.

[34] F. Melo, Receiver Operating Characteristic (ROC) Curve, Springer, New York, NY, USA, 2013. 